\title{
Le rôle du corps dans la pédagogie d'Alain
}

\section{Caroline Pigno-Richard}

\section{OpenEdition \\ Journals}

Édition électronique

URL : https://journals.openedition.org/leportique/894

DOI : 10.4000/leportique.894

ISSN : $1777-5280$

\section{Éditeur}

Association "Les Amis du Portique"

\section{Référence électronique}

Caroline Pigno-Richard, «Le rôle du corps dans la pédagogie d'Alain », Le Portique [En ligne], e-Portique, mis en ligne le 08 janvier 2007, consulté le 11 septembre 2022. URL : http://journals.openedition.org/ leportique/894 ; DOI : https://doi.org/10.4000/leportique.894

Ce document a été généré automatiquement le 11 septembre 2022.

Tous droits réservés 


\title{
Le rôle du corps dans la pédagogie d'Alain
}

\author{
Caroline Pigno-Richard
}

«Car si quelqu'un se montre insensible aux arts, ou rétif devant la géométrie, on ne peut s'en consoler que si on le méprise. C'est demander

trop, peut-être ; mais on n'a pas le droit de demander moins. Le plus beau est, qu'avec toute la sévérité possible, on ne peut forcer, puisque c'est le libre qu'on veut. ${ }^{1}$.

1 La culture ne se réduit pas à la somme des savoirs que l'on possède. Elle se définit par la manière dont on les intègre et les utilise. On peut donc y voir le but, non seulement de l'enseignement, mais également de l'éducation, en tant que celle-ci est une formation de tout l'être.

2 Plusieurs courants ont rejeté l'idée même de culture. Le courant révolutionnaire, ou gauchiste, voyait dans la culture une forme d'intégration à la société bourgeoise et dénonçait la répression par le savoir. Puis le courant pragmatiste a rejeté un enseignement "désintéressé ", donc inutile, au profit d'une formation technique et civique, adapté aux besoins de l'époque. Enfin, le courant de la pédagogie libertaire, ou non directive, a rejeté les modèles imposés, pour favoriser la spontanéité et la créativité de l'enfant. Alain lui, défend cette idée de culture tout au long des Propos sur l'éducation. Si la culture est formation, plus qu'information, elle doit s'appuyer sur les deux disciplines qui sont vraiment formatrices, la poésie et la géométrie. L'une est l'essentiel des lettres, l'autre le fondement des sciences exactes. Sans géométrie, l'homme reste prisonnier de ses préjugés et de ses rêves. Sans poésie, l'esprit s'avère incapable de comprendre et de dominer ses passions. Complémentaires, elles sont opposées, la géométrie donnant à l'enfant la clé du monde extérieur, la poésie, celle du monde humain.

3 Alain reprend Comte en ce qui concerne l'ordre d'apprentissage de ces enseignements. L'enseignement doit être littéraire d'abord, surtout à l'école primaire où le but est 
l'acquisition d'une langue bien faite. Mais, considérant la mentalité enfantine et son fonctionnement, Alain montre que l'enfant va en réalité des signes aux choses, de la magie à la science. Et il est dangereux de ne pas prendre l'enfant comme il est. Le rôle du maître est donc de lui enseigner les signes avant les choses, la politesse avant la vérité, la poésie avant l'idée, l'expression correcte avant la pensée juste. Permettre à l'enfant d'obtenir une réelle maîtrise de lui-même est la finalité du maitre. L'éducation littéraire est trop intellectuelle pour assurer cette maîtrise. C'est pourquoi, à l'instar des Grecs, Alain associe toujours la gymnastique à la musique, comme formant l'infrastructure et l'élément premier de l'éducation.

L'enfance : monde de l'imagination et des passions

4 Le monde de l'enfant est avant tout pour Alain celui du corps. Au premier âge, la vie du nourrisson n'est réglée qu'au rythme de ses repas et de ses besoins que nous pourrions qualifier de besoins primaires ou animaux. L'enfant «(...) juge des choses et des hommes d'après ses propres affections, ou d'après les mouvements de son propre corps. $»^{2}$ L'enfant est donc totalement dépendant de son corps, ce n'est que par lui et à travers lui qu'il acquiert une première connaissance du monde extérieur. Toute sa pensée est réglée en fonction de ses émotions et de ses réactions corporelles. "L'état d'enfance est toujours caractérisé par ceci que l'émotion l'emporte »" émotion en tant que « (...) mouvement dans le corps, mouvement dépendant principalement de la structure et des actions environnantes. $»^{4}$.Car l'enfant découvre les choses d'abord par ses sens et notamment par la vue :

«L'idéalisme est un état d'enfance; et le profond Maine de Biran rattache cette étrange opinion à l'exercice du sens de la vue. L'œil est idéaliste. Et pourquoi ? Parce que les impressions de l'œil se font sans aucun travail, sans aucune réelle exploration. $»^{5}$

5 Parce que l'enfant reçoit, par la vue, de manière totalement passive, les informations sur les choses et le monde extérieur, il ne peut qu'être dans l'erreur. Toute passivité dans la connaissance ne peut justement amener à la connaissance véritable.

De même, l'œil ne donne à l'enfant qu'une suite d'images sans consistance, d'autant plus trompeuses qu'elles représentent sa seule expérience du monde, et qu'elles dépendent essentiellement du caprice des adultes qui allument ou éteignent la lumière, changent le spectateur de place, changeant ainsi tout le spectacle. Les sens sont trompeurs et l'enfant en est totalement dépendant. Car il n'y a pas que la vue qui trompe l'enfant dans ses premières perceptions du monde, l'ensemble de ses sens en est responsable :

«Je comparerais toute la surface sensible de l'enfant à une sorte d'œil qui est comme un théâtre ou un écran, successivement occupé par des ombres agréables ou désagréables qui sont toutes des visions c'est-à-dire des choses reçues ou subies. ${ }^{6}$

7 Tout ce qui est donc pensée dans l'enfant est étroitement lié à un état de dépendance corporelle. Celle-ci est, pour Alain, la marque de l'imagination : « L'enfant est d'abord tout imagination. $»^{7}$. Imaginer, c'est, selon l'idée de Descartes, penser en fonction des états et des mouvements du corps. Par opposition à l'entendement, qui juge les choses telles qu'elles sont, indépendamment de nous, l'imagination nous donne une représentation du monde conforme à nos affections. Cette imagination, reine de l'enfance est une grande source d'erreur car elle nous donne une image du monde conforme à nos affections et non aux choses telles qu'elles sont réellement : 
«Car si l'on entend ce mot selon l'usage, l'imagination n'est pas seulement, ni même principalement, un pouvoir contemplatif de l'esprit, mais surtout l'erreur et le désordre entrant dans l'esprit en même temps que le tumulte du corps. $»^{8}$. dépasser ce stade de l'imaginaire. L'imagination explique, par ce fait, l'égocentrisme dont fait preuve l'enfant : ses désirs et ses envies sont pris pour des réalités. Il imagine que tout ce qu'il subit corporellement existe. Il est donc un être de passion, au sens où «elles correspondent au premier rapport qui s'établit entre les émotions comme telles (mécanisme) et le jugement (ou la conscience) qui les observe, les rappelle et les annonce. La crainte est déjà une passion. »'. L'enfant est esclave parce qu'il est passionné, ou plutôt parce qu'il est privé de liberté. Or cette réalité qui lui est propre ne peut se réaliser que par le biais des individus qui sont autour de lui. Il n'y a pas de choses existantes, il n'y a que des gens, et ceci caractérise ses premières pensées : "Les choses sont regardées et traitées comme des personnes. $»^{10}$.

9 Les affections du corps rendent ainsi l'enfant totalement passif dans ses jugements et font de lui un être de pures passions, réactif dans ses actes qui ne sont que peur, colère et désespoir. L'enfant est donc incapable au départ, de saisir le monde réel, il est condamné à l'imagination et ne voit les choses que selon ses affections et ses préjugés. Or, pour devenir adulte, l'enfant a besoin de se libérer de l'emprise des passions afin de parvenir à une maîtrise volontaire de son corps et ainsi développer son entendement. La pensée doit pouvoir prendre le pas sur le corps. Et la première étape de cette réconciliation entre l'âme et le corps est l'habitude.

10

L'habitude n'est pas chez Alain, comme chez beaucoup de philosophes, une aliénation, elle est simplement le contraire de l'émotion. Alors que dans l'émotion l'enfant perd ses moyens, c'est-à-dire le contrôle de son corps, il acquiert, avec l'habitude, ce contrôle, cette maîtrise de soi sans laquelle aucun acte volontaire n'est possible. L'habitude s'oppose également chez Alain à la coutume. Celle-ci n'est que routine acquise passivement et montée en chacun comme un mécanisme rigide. Elle peut alors devenir nuisible dès lors qu'elle est dérangée : l'enfant ou l'individu se retrouvant «hors de lui ", la coutume instaurée laisse place à l'émotion violente. Elle est ainsi le costume derrière lequel s'abrite le timide et le paresseux et qui endort toutes nos pensées. Et le plus dangereux sans doute est que non seulement elle fait agir, mais elle fait également penser : la plupart de nos croyances et de nos «principes » sont de cet ordre : ils font partie de la coutume. Or, contrairement à elle, l'habitude est acquise activement et elle permet d'apprendre: apprendre n'étant pas ici acquérir quelque chose de nouveau, mais vaincre l'obstacle présent en chacun. En divisant la difficulté et en éliminant tous les gestes parasites, l'enfant parvient à supprimer l'agitation dûe à la peur et à la timidité. Tout essai commence dans la maladresse. C'est cela qu'il faut vaincre. Avoir une habitude est donc tenir son corps et faire ce qu'on veut, ou du moins savoir ce qu'on veut.

11 Ainsi, alors que la coutume provoque une certaine jouissance toute passive, celle de la paresse et de la sécurité, l'habitude difficile est par essence une joie: celle de la puissance souveraine sur son corps qui accompagne l'acte réussi. L'habitude réalise de ce fait l'indépendance de l'esprit et cette possession de soi qu'elle permet est la condition même de la volonté, élément indispensable à tout apprentissage et éducation véritable. Aussi, la première finalité de l'éducation est-elle de réconcilier l'âme avec le 
corps, et pour ce faire, Alain évoque une éducation du sentiment, inséparable de la pensée.

La pensée comme réconciliation de l'âme et du corps

«Le principe de la morale est dans le catéchisme, c'est que l'homme est composé

d'un corps et d'une âme. »"11

La faute est alors de céder aux automatismes du corps et de se laisser aller. Mais, plus subtilement encore, la faute consiste à donner un sens à ces automatismes, à chercher quelque esprit dans notre animal machine. Certes, dans la peur, la faim, la colère, la douleur, les larmes, le rire, la jalousie, l'amour, nous apprenons notre intime dépendance envers cette machine humaine qui se meut et s'agite sans notre permission. Mais il n'y a aucune âme, même inférieure, dans ces automatismes. La fatalité des passions est dans ce mauvais mélange du corps et de l'esprit, « où la pensée est comme une pièce sourde et aveugle dans un mécanisme, et où, en revanche, les forces mécaniques participent à cette suite, de cette obstination et même de ces ruses qui sont propres à la pensée. $\rrbracket^{12}$ Or ce mauvais mélange n'existe que parce que l'individu le veut bien. Il faut donc renvoyer les passions à l'ordre des choses, comprendre que le mouvement de fuite est aussi mécanique que les mouvements du cœur, et ainsi chacun sera capable de manier ses passions comme des forces mécaniques. Alain le montre dans ses Eléments de philosophie : notre corps n'est qu'un cadavre. Vivre c'est trainer ce cadavre et ainsi nous mourrons lorsque c'est lui qui nous traîne ${ }^{13}$. Mais le corps n'est pas que pure mécanique et expression des passions, il est également, le lieu du sentiments, ou des sentiments. Et si Alain se méfie de ceux - les psychologues et les psychanalystes -, qui excusent les comportements déviants par la présence en chacun d'un inconscient incontrôlable, il ne récuse pas l'importance de ces sentiments sur les actions humaines.

Pour Alain, le sentiment, loin de suivre l'intelligence, la précède, car une pensée qui ne naît pas d'un sentiment n'est pas vraiment ma pensée. Il n'est pas un état d'âme naissant des humeurs de l'individu mais il est au contraire émotion pure et simple, émotion du corps sauvée et transfigurée par la plus haute volonté. « La pensée n'est point destinée à régner mais à servir. (...) Pour décider, il faut de la force; la raison n'a jamais que de la lumière. $»^{14}$ Cette force est celle du sentiment dont Alain donne une définition réelle: "C'est le plus haut degré de l'affection (...). Par exemple, la peur est une émotion ; la lâcheté est une passion. Le sentiment correspondant est le courage. Tout sentiment se forme par une reprise de volonté (ainsi l'amour jure d'aimer). Et le sentiment fondamental est celui du libre arbitre (ou de la dignité, ou de la générosité, comme dit Descartes). Ce sentiment a quelque chose de sublime $q u$ 'on retrouve dans les sentiments particuliers. $»^{15}$ Certes nos sentiments dépendent des circonstances et doivent trouver appui dans l'inférieur. Mais le plus important est de juger et non pas de contempler ses sentiments et de les attendre comme quelque chose venu du ciel. Nos sentiments doivent être portés à bras, ce qui signifierait que l'enfant n'a pas de sentiments. Mais si, l'enfant a des sentiments plus fragiles mais non moins profonds: outre les attachements familiaux et sociaux, il existe en effet chez tout enfant sain le sentiment qui correspond à sa volonté constante de grandir et de se dépasser. Ce sentiment est l'admiration.

14 Le plus important, là encore, dans le sentiment est la volonté. Mais celle-ci ne peut rien sans l'habitude qui rend le corps disponible : il faut apprendre à sentir et l'on ne sent rien sans l'avoir appris. Et, plus encore, la volonté et l'habitude ne suffisent pas :

«Au niveau du sentiment, on prétend sentir comme on veut, et certes on y arrive jamais. Ce qui reste, dans le sentiment, d'émotion et de passion, surmontées mais frémissantes, est la matière du sentiment. Exemple: la peur dans le courage, le 
désir dans l'amour, l'horreur des plaies dans la charité. On aperçoit que le sentiment est la source des plus profondes certitudes. $»^{16}$

\section{contes et dans les jeux, puis dans la poésie, la musique et le théâtre. Tout ce qui peut} participer à cet apprentissage des sentiments est bon pour l'enfant. Car de cet apprentissage, il en ressortira sa conscience et son libre arbitre. Sentir, c'est être soi et penser par soi. La valeur du sentiment est que le corps y porte la pensée, et celle-ci n'est plus une idée abstraite et impersonnelle, elle appartient pleinement à l'individu. Et Alain critique par là une grossière erreur des pédagogies modernes qui remplit les esprits d'idées d'emprunt et fait des pensées sans penseurs. Il faut au contraire selon lui cultiver non pas l'esprit séparé, mais bien le sentiment, c'est-à-dire la pensée unie au corps, enracinée. Tout comme l'habitude - et grâce à elle -, le sentiment est réconciliation de l'âme et du corps. D'où l'importance en pédagogie de ne jamais en privilégier un plutôt que l'autre.

17

Cette idée peut paraître contradictoire chez un penseur où l'instruction et l'apprentissage des Humanités a autant d'importance dans l'éducation des enfants. Mais il faut comprendre qu'il y a chez Alain, des degrés dans l'apprentissage, et des fondations à assurer avant d'apporter à l'enfant la Culture dont il aura besoin pour se forger. Partant de sa psychologie propre et de son mode de fonctionnement, si le maître ne lui donne pas les moyens de dépasser l'univers des passions et des affections qui le tiraillent sans cesse, afin de développer la volonté et l'habitude, nécessaires à la " conquête » du sentiment qui lui permettra de se connaître lui-même, et même d'être véritablement lui-même, l'enfant ne pourra jamais développer sa conscience. Pire, il ne sera jamais en mesure d'apprendre véritablement. La vraie culture est d'accorder ce qui comprend avec ce qui sent. Sentiment et pensée sont inséparables. Et, suivant Platon, Alain propose, en complément de cette éducation des sentiments, une éducation du corps, par la gymnastique, qui doit permettre à l'enfant d'apprendre à se dominer et à ne pas se laisser déborder par ses affections.

L'importance de la gymnastique dans l'éducation

La gymnastique, qui comprend non seulement l'éducation physique, mais aussi tout ce qui permet d'acquérir de bonnes habitudes - écriture, danse, politesse...-, a pour but de dénouer le corps, de le délivrer de la violence des passions, source de toute maladresse. Elle donne une habileté à l'enfant, en ce sens qu'elle permet de faire un mouvement sur commande, sans contractures inutiles. L'enfant acquiert l'aisance qui fait de l'organisme son instrument silencieux. La gymnastique crée ces habitudes qui font du corps de l'enfant, son corps. Elle est déjà culture en ce qu'elle délivre de "la coutume » et donne à l'enfant l'aptitude de faire face à toute situation, la disponibilité : «Ce sont ces nœeuds, fils de colère, que poursuit la véritable gymnastique qui, par l'assouplissement du corps, prépare l'action volontaire. La gymnastique est l'école de la volonté. Le but à atteindre est de faire exactement ce qu'on veut, sans rien de plus. ${ }^{17}$. Sans la gymnastique, l'homme n'est jamais qu'un pédant, pensée savante dans une bête inéduquée, peureuse et violente, qui sans cesse le trahit et se venge. 
19 Néanmoins, elle n'est qu'une partie de l'éducation. L'athlète qui n'est qu'athlète n'est qu'un pédant retourné. Sa pensée est comme diffuse dans un corps qu'elle domine sans pouvoir se reprendre et réfléchir :

«Le pédant nous fait voir un esprit bien fait dans un corps maladroit. L'animal a été oublié, et se venge par un désaccord entre l'enveloppe et le contenu, qui se sent dans la moindre parole. L'athlète est tout le contraire d'un pédant, parce que le corps a reçu tous les soins et toute la culture possible; et, comme toutes les actions de l'athlète sont justes et belles, on voudrait dire qu'il pense bien, si ce n'était que sa pensée est comme répandue en son corps au lieu d'être rassemblée dans le discours. Ce n'est point l'homme, c'est un pédant retourné. Il n'est que forme extérieure ; pour les autres et non pour soi. On le veut statue. $\aleph^{18}$.

L'homme cultivé n'est ni l'athlète, ni le pédant, il est l'homme complet, dont le corps et l'esprit sont unis dans un seul et même style. La gymnastique ne change pas la nature de l'homme, elle la délivre parce qu'elle est d'abord un exercice de la volonté, c'est-àdire un apprentissage de la maîtrise de soi : «Le gymnase est l'école de la volonté. ${ }^{19}$. Il n'y a pas d'action libre sans une parfaite disposition de soi: "Le corps, non formé par gymnastique s'emporte aussitôt. $\aleph^{20}$. La gymnastique n'est pas uniquement un exercice corporel. L'intime union de l'âme et du corps fait qu'elle acquiert une valeur morale et qu'il arrive que "Le maître de philosophie vous renvoie au maître de gymnastique. $»^{21}$. Il est essentiel, pour la vie morale, que l'enfant soit maître de ses pensées, et s'il ne pense pas comme il veut, grâce à la gymnastique, il parvient à agir comme il veut. Mais comme les pensées sont toujours liées, plus ou moins, aux mouvements du corps, et qu' " (...) on ne peut pas du tout penser le son $i$ en ouvrant la bouche. ${ }^{22}$, nous avons sur nos pensées, sinon un pouvoir direct du moins un pouvoir indirect, par l'intermédiaire des attitudes. Si bien que « (...) c'est (...) par la gymnastique d'abord que la pensée réduit les passions. »"

21 La gymnastique est donc primordiale en pédagogie si l'on pense aux conditions de l'attention véritable. Alain distingue l'attention vraie de l'attention facile. Mais il existe une autre distinction, non moins importante, entre l'attention nouée, ou intempérante, et l'attention déliée ou libre. Le maître croit souvent que l'élève est attentif parce qu'il le voit immobile à son banc, fronçant les sourcils et tendant l'oreille, mais «(...) les signes de l'intelligence sont bien trompeurs. Front soucieux, regard pénétrant, bouche tordue par les discours contraires, cela annonce plus de pensée que l'homme n'en peut conduire. $\gg^{24}$, et ainsi se montre l'attention intempérante. Mais « (...) cette attention immobile trompera toujours $»^{25}$. Les nœuds que l'homme forme dans son corps étranglent ses pensées. Cette tension, qui fatigue sans profit, n'est pas à prendre pour travail ou effort. Il n'y a d'attention véritable que celle qui est du premier et frais moment, car il est impossible, même pour l'adulte, d'être attentif très longtemps. Aussi faut-il nommer « (...) attention déliée cette simple, libre et puissante modestie que l'on remarque dans les bons écoliers. $»^{26}$. Elle est la seule attention authentique. La gymnastique est ce qui permet d'apprendre à se délier ainsi ou par poésie car "L'objet beau délie $»^{27}$. La gymnastique, par là, joue dans l'éducation un rôle analogue à celui des humanités. En ce sens, il faut redire, avec Platon, que gymnastique et musique sont l'essentiel de l'éducation :

«L'art grec n'a rien de mystérieux ni d'effrayant. Rien n'y est démesuré, tout y est en ordre, soit dans le temple, soit dans l'homme. L'animal humain n'y fait point honte, et n'a nullement honte. Il atteint la perfection qui lui est propre, et se borne là, par un bonheur de forme qui n'a jamais été surpassé. Ces beaux corps signifient gymnastique et musique ensemble, ce qui veut dire que, des orteils aux cheveux, ils sont tout entiers pensée et volonté. $»^{28}$. 

maîtriser lui-même, que ce soit au travers de ses gestes ou au travers de ses pensées. La culture est ce qui délie l'enfant, et n'est pas qu'une somme de savoirs. Elle libère l'enfant de ses passions et de ses préjugés. «La gymnastique est pour le corps et la musique pour l'âme. ${ }^{29}$ La musique est pour Alain l'âme de la culture. Il y inclut non seulement le chant et l'étude des instruments, mais aussi la culture des muses, la danse, la poésie, le drame. Il faut offrir à l'enfant de beaux modèles. La musique est la culture littéraire avant tout. Par sa musique, par le son, le nombre et le rythme, la poésie dispose le corps et transfigure les émotions. A l'encontre de la musique, elle est déjà pensée, son chant a un sens. Il faut réciter avant de comprendre, commencer par des vers difficiles dont le sens échappe. L'enfant aime répéter des mots dont le sens lui échappe. Nous le voyons par exemple avec les comptines. Il a un besoin spontané de rythme, de rime, de sonorités. Avant de vouloir donner du sens aux choses, il faut pouvoir se mettre en état de penser. Et cet état de penser est tout ce que le maître doit viser lorsqu'il éduque un enfant. Aussi la musique participe t-elle à ce «dressage » du corps au même titre que la gymnastique car elle donne des habitudes à l'enfant qui apprend ainsi à dominer son corps par l'écoute.

Conclusion

Alain, dans sa pédagogie même affirme une triple unité : unité du corps, unité de la pensée, et unité de l'homme qui pense avec tout le corps. A partir de cette affirmation, il est impossible pour le maître de ne s'intéresser, dans l'éducation des enfants, qu'au développement de l'esprit. Car l'esprit n'est rien sans le corps, ou plutôt, il ne permet rien, aucun apprentissage véritable, si, conjointement, l'enfant n'apprend pas à maitriser les affections qui bouleversent sans cesse son corps. A ce propos, Alain rejoint Descartes sur les rapports existants entre l'âme et le corps : l'union entre un pur agir et un pur être.

"L'âme c'est ce qui refuse le corps. Par exemple ce qui refuse de fuir quand le corps tremble, ce qui refuse de frapper quand le corps s'irrite, ce qui refuse de boire quand le corps a soif, ce qui refuse de prendre quand le corps désire, ce qui refuse d'abandonner quand le corps a horreur. Ces refus sont des faits de l'homme $» .^{30}$

La conscience pour Alain est un acte, elle est réflexion, attention, et il ne peut y avoir pensées que lorsque nous les formons par notre réflexion. La vraie pensée est donc une pensée consciente, tout le reste renvoie à notre corporalité. Seul le corps est véritablement, même l'esprit est un "éternel absent " ${ }^{31}$. Tout ce qui se joue en nous n'est que le fruit des rapports existant entre notre pensée et le monde extérieur, «La pensée n'est pas plus en moi que hors de moi, car le hors de moi est pensé aussi, et les deux toujours pensés ensembles. $\aleph^{32}$. La pensée est donc partout et nulle part à la fois. L'intérieur n'existe qu'en tant qu'il est extériorisé. L'homme ne pense que parce qu'il perçoit : « ... Un homme qui ne perçoit point n'est pas livré à ses propres idées; bien plutôt il n'a plus d'idée; il dort. S'il rêve, c'est que l'univers extérieur l'attaque par quelque côté; c'est qu'il commence à percevoir. " $^{33} 34$

Nombreux sont les psychologues et les psychanalystes, très présents en pédagogie aujourd'hui, qui critiquent Alain en opposant sa vision de l'unité entre l'âme et le corps, à l'existence d'un inconscient corporel. Chaque fois que nous cessons d'être attentif, des signes extérieurs du corps apparaissent sans que nous y prêtions attention : par exemple le fait de se mettre à remuer la main. Mais pour Alain, ce geste est loin d'avoir une signification puisqu'il est hors du champ de nos pensées. Le reproche qu'il fait alors 
aux psychologues est de chercher, en définitive, de la signification là où il n'y a que du pur mécanisme. La pensée est partout où elle est un acte volontaire. Sans volonté, pas de conscience et donc pas de pensée. Il apparait alors comme fou de s'intéresser à des signes pour leur donner des significations qui n'ont aucun lieu d'être. La seule préoccupation valable dans le domaine pédagogique est cette réconciliation chez l'enfant, entre d'un côté ses passions et le corps qui les abritent et les nourri, et de l'autre sa pensée, volontaire et assumée. Et nous pouvons constater que nous sommes loin des questions qui traversent la réflexion éducative aujourd'hui où le seul problème semble être de savoir quelle culture enseigner pour quelle société. Sans doute est-ce parce que l'enfant n'apprend plus à se connaître et à se dominer, qu'il ne parvient pas à trouver sa place au sein de la société dans laquelle il évolue.

\section{BIBLIOGRAPHIE}

Platon, La république, Paris : Flammarion, 2002, 801 p.

Alain, Les arts et les dieux, Gallimard, Paris : Bibliothèque de la Pléiade, 1958, 1440 p.

Alain, Propos I, Gallimard, Paris : Bibliothèque de la Pléiade, 1956, 1370 p.

Alain, Propos II, Gallimard, Paris : Bibliothèque de la Pléiade, 1970, 1326 p.

Alain, Propos sur le bonheur, Paris : Gallimard, 1928, 217 p.

Alain, Propos sur l'éducation suivis de Pédagogie enfantine, Paris : PUF, 1986, 384 p.

Alain, Eléments de philosophie, Paris : Gallimard, 1941, 378 p.

Chateau R., La philosophie par les textes, Tome I

\section{NOTES}

\section{Notes}

Alain, Propos du 18 avril 1927, in Propos I, p 704.

2. Alain, Pédagogie enfantine, $7^{\text {ème }}$ leçon, p 251.

3. Ibid., $2^{\text {ème }}$ leçon, p 232.

4. Ibid., p 231.

5. Alain, Préliminaires à la mythologie, in Les arts et les dieux, p 1123.

6. Alain, Préliminaires à la mythologie, in Les arts et les dieux, p 1106.

7. Alain, Pédagogie enfantine, Septième leçon, p 251.

8. Alain, Le système des beaux-arts, livre $1^{\mathrm{er}}$, in Les arts et les dieux, pp 221-225.

9. Alain, Pédagogie enfantine, p 236.

10. Alain, Préliminaires à la mythologie, in Les arts et les dieux, p 1141.

11. Alain, Propos du 26 mai 1924, in Propos I, p .

12. Alain, Idées, $\mathrm{n}^{\circ} 148$ et suivants.

13. Alain, Eléments de philosophie, pp 68-71. 
14. Alain, Les idées et les âges, $n^{\circ} 318$.

15. Alain, Définitions, in Les arts et les dieux, p 1088.

16. Ibid.

17. Cours inédit de 1925-26, cité par Chateau R., in La philosophie par les textes, Tome I, p 129.

18. Alain, Propos du 18 avril 1924, in Propos I, pp 594-595.

19. Cours inédit de 1925-26, cité par Chateau R., p 129.

20. Alain, Propos sur le bonheur, LXIX, 27 décembre 1921, pp 160-161.

21. Ibid., XVII, 16 mars 1922, p 48.

22. Ibid., XVIII, 24 décembre 1913, p 50.

23. Alain, Propos sur la religion, IV, du 31 janvier 1914.

24. Alain, Minerve, VII.

25. Alain, Propos sur l'éducation, LX, p 152.

26. Alain, Esquisse de l'homme, LXV, du 5 décembre 1929.

27. Alain, Minerve, VIII.

28. Alain, Préliminaires à la mythologie, in Les arts et les dieux, p 1148.

29. Platon, République, II, $376 \mathrm{e}$.

30 Alain, Définitions in, Les arts et les dieux, p 1031.

30.

31. Alain, Les dieux in, Les arts et les dieux, La Pléiade p 1321.

32. Alain, Eléments de philosophie, p 201.

33.

34. Alain, Propos, 23 septembre 1921, in Propos I, pp 298-299.

\section{RÉSUMÉS}

Lorsque l'on s'interroge sur une " éducation idéale » possible, le meilleur résumé est sans doute "un esprit sain dans un corps sain ». Or cette maxime peut surprendre dans un système éducatif où tout est basé sur l'instruction et l'importance des savoirs encyclopédiques, nécessaires au bon développement de l'individu. Pourtant, certains auteurs, comme Alain, sont parvenus à trouver une forme de compromis entre, d'un côté l'importance du savoir pour la formation de l'esprit critique et le développement du jugement critique, et d'un autre côté la nécessité, tout aussi importante, d'un développement harmonieux et concomitant du corps de l'enfant pour parvenir à une éducation complète et utile pour la société.

When one wonders about a possible « ideal education » no doubt the best summary would be «a healthy spirit in a healthy body». But this maxim can surprise in an educative system where everything is based on instruction and the importance of an encyclopaedic knowledge being required for good development of the individual. Yet certain authors, as Alain, came to fund a compromise between the importance of knowledge to form a spirit criticism and development of critical judgement on one side and the equally important need for a harmonious and concomitant development of the child's body to reach a complete and useful education for society. 


\section{AUTEUR}

\section{CAROLINE PIGNO-RICHARD}

Chargée de cours à l'université Paul Valéry de Montpellier, et Docteur en Sciences de l'éducation depuis novembre 2004, spécialiste d'Alain, Caroline Pigno-Richard fait partie depuis deux ans du CERFEE (Centre de Recherches sur la Formation, l'Education et l'Enseignement) où elle poursuit ses recherches dans le domaine de l'éducation et plus généralement dans celui de l'histoire des idées éducatives. Parmi les articles déjà parus : L'autorité chez Alain : contraindre pour mieux libérer, in Cerfee $n^{\circ} 21$, à paraître sept. 2006, La philosophie de l'éducation d'Alain : la pédagogie pensée comme une anthropologie, Actes du colloque international de l'UCO, juin-juil. 2005, Quelle place pour l'éducation des adultes dans le système éducatif français ? Entre formation populaire et éducation permanente, Actes du colloque international du REF, sept. 2005, Condorcet, Jules Ferry et Alain : une histoire de l'école de la République, Actes du Colloque du Congrès de l'AECSE, sept. 2003, De la politique à la pédagogie : problématisation des finalités éducatives dans les propos d'Alain, in Penser l'éducation, $n^{\circ} 18$, Emergences Ed., déc. 2005, Peut-on encore enseigner la morale en France? Réflexions à partir de l'exemple de la Belgique. In Diotime l'Agora, à paraître. 